\title{
Increased Concomitant Drug Peak Blood Concentration
}

National Cancer Institute

\section{Source}

National Cancer Institute. Increased Concomitant Drug Peak Blood Concentration. NCI

Thesaurus. Code C54610.

Increased maximum (peak) concentration of a concomitant drug observed after its administration. 